\title{
WILLIAM PETTY
}

\section{Schriften}

zur politischen Ökonomie

und Statistik 


\section{ÖKONOMIEHISTORISCHE TEXTE}

HERAUSGEGEBEN VON

\section{WILLY GÖRLICH}

IM AUFTRAG

DES ZENTRALINSTITUTS

FÜR WIRTSCHAFTSWISSENSCHAFTEN

DER AKADEMIE DER WISSENSCHAFTEN

DER DDR 


\section{WILLIAM \\ PETTY}

Schriften

zur

politischen Okkonomie

und

Statistik

Übersetzt und mit einem Nachwort herausgegeben von

WILLY GÖRLICH

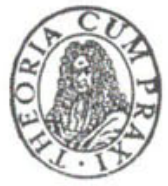

AKADEMIE - VERLAG BERLIN

1986 
Die Übersetzung beruht auf der englischen Ausgabe:

The Economic Writings of Sur William Petty, ed. by C. H. Hull, Cambridge 1899

Umschlagbild :

J. Smith, Bildnis William Petty Staatliche Kunstsammlungen Dresden

ISBN 3-05-000 029-5

ISSN 0233-0946

Erschienen im Akademie-Verlag, DDR - 1086 Berlin,

Leipziger Str. 3-4.

(c) Akademie-Verlag Berlin 1986

Lizenznummer: $202 \cdot 100 / 197 / 85$

Printed in the German Democratic Republic

Gesamtherstellung: VEB Druckere "Gottfried Wilhelm Leibniz", 4450 Gräfenhainichen $\cdot 6341$

Lektor: M. Stappenbeck

Schutzumschlag- und Einbandgestaltung: Eckhard Steiner

LSV 0306

Bestellnummer: $7543184(2093 / 10)$

002800 